\title{
A novel Plasmodium vivax vaccine based on recombinant chimpanzee adenovirus ChAd63 and MVA expressing TRAP
}

\author{
Karolis Bauza', Oliver Billker ${ }^{2}$, Adrian VS Hill ${ }^{1}$, Arturo Reyes-Sandoval ${ }^{1 *}$ \\ From Challenges in malaria research \\ Basel, Switzerland. 10-12 October 2012
}

\section{Background}

P. vivax is the most geographically widespread human malaria and is considered to be the most prevalent form in some regions of Latin America, Central and South-East Asia, accounting for up to 390 million clinical infections every year and an estimated 2.6 billion people being at risk of infection with $P$. vivax $[1,2]$. An effective vaccine against this protozoan would have a major global impact on the disease burden [3]. Modified Vaccinia Ankara (MVA) and the chimpanzee adenovirus ChAd63 are two clinically relevant viral vectors that have been shown to induce strong and protective antibody and T-cell responses against $P$. falciparum TRAP, both in pre-clinical studies and clinical trials [4-7].

\section{Materials and methods}

We developed recombinant ChAd63 and MVA vectors expressing P. vivax TRAP (PvTRAP), which were used to assess $\mathrm{T}$-cell and antibody responses upon sequential immunisation (prime-boost) of mice. Vaccine efficacy was assessed through challenge with a newly developed transgenic $P$. berghei expressing PvTRAP.

\section{Results}

High antibody titres and frequencies of PvTRAP-specific $T$ cells were induced in all tested inbred and outbred mouse strains. The newly developed parasite showed similar fitness to wild type P. berghei and was successfully used to infect and assess protection in vaccinated mice. The Ad-MVA prime-boost regimen induced good protective levels regardless of the mouse strain.

'The Jenner Institute, University of Oxford, Oxford, Oxfordshire, OX3 7DQ, UK

\footnotetext{
Full list of author information is available at the end of the article
}

\section{Conclusions}

The strong immunogenicity and protective efficacy elicited by the recombinant ChAd63 and MVA viruses expressing PvTRAP indicate that this vaccine approach has a good potential to be tested in clinical trials in the near future.

\section{Acknowledgments}

A.R-S and A.V.S Hill are Jenner Investigators. This work was supported by a Wellcome Trust Career Development Fellowship to A.R-S.

\section{Author details}

'The Jenner Institute, University of Oxford, Oxford, Oxfordshire, OX3 7DQ, UK. ${ }^{2}$ Wellcome Trust Sanger Institute, Hinxton, Cambridge, Cambridgeshire, CB10 1SA, UK.

Published: 15 October 2012

\section{References}

1. Guerra CA, et al: The international limits and population at risk of Plasmodium vivax transmission in 2009. PLoS Negl Trop Dis 2010, 4(8): e774.

2. Price RN, et al: Vivax malaria: neglected and not benign. Am J Trop Med Hyg 2007, 77(6 Suppl):79-87.

3. Hay SI, Guerra CA, Tatem AJ, Noor AM, Snow RW: The global distribution and population at risk of malaria: past, present, and future. Lancet Infect Dis 2004, 4(6):327-336.

4. O'Hara GA, et al: Clinical assessment of a recombinant simian adenovirus ChAd63: a potent new vaccine vector. J infect Dis 2012, 205(5):772-781

5. Reyes-Sandoval A, et al: Prime-boost immunization with adenoviral and modified vaccinia virus Ankara vectors enhances the durability and polyfunctionality of protective malaria CD8+ T-cell responses. Infect Immun 2010, 78(1):145-153.

6. Reyes-Sandoval A, et al: Single-dose immunogenicity and protective efficacy of simian adenoviral vectors against Plasmodium berghei. European journal of immunology 2008, 38(3):732-741.

7. Reyes-Sandoval A, et al: CD8+T effector memory cells protect against liver-stage malaria. J Immunol 2011, 187(3):1347-1357.

doi:10.1186/1475-2875-11-S1-049

Cite this article as: Bauza et al: A novel Plasmodium vivax vaccine based on recombinant chimpanzee adenovirus ChAd63 and MVA expressing TRAP. Malaria Journal 2012 11(Suppl 1):O49. 